\title{
Perfil da mortalidade infantil a partir da investigação de óbitos
}

\author{
Child mortality profile based on investigation of obituary records
}

Perfil de la mortalidad infantil a partir de la investigación de fallecimientos

Camila Marques Careti ${ }^{1}$, Ana Helena Parra Scarpelini ${ }^{2}$, Maria Cândida de Carvalho Furtado ${ }^{3}$

${ }^{1}$ Discente do curso de graduação em Enfermagem da Escola de Enfermagem de Ribeirão Preto da Universidade de São Paulo (EERP/USP). Ribeirão Preto, SP, Brasil. E-mail: camilamarquesc@hotmail.com.

2 Médica pediatra. Médica da Secretaria Municipal de Saúde de Ribeirão Preto. Ribeirão Preto, SP, Brasil. E-mail: anahelenapa@yahoo.com.

${ }^{3}$ Enfermagem, Doutora em Enfermagem em Saúde Pública. Professora Doutora da EERP/USP. Ribeirão Preto, SP, Brasil. E-mail: mcandida@eerp.usp.br.

\section{RESUMO}

O estudo objetivou analisar o perfil da mortalidade infantil de residentes em Ribeirão Preto -SP, entre 2009 e 2011 , a partir dos dados do Comitê de Mortalidade Materno-Infantil. No período, ocorreram 224 óbitos, sendo 58 evitáveis. Crianças com menos de uma semana de vida, baixo peso ao nascer e idade gestacional inferior a 30 semanas foram as que mais morreram; o pré-natal foi considerado insuficiente para a maioria dos casos. Houve associação entre idade do óbito e idade gestacional; ter mais anos de estudo demonstrou um efeito protetor com relação ao óbito na primeira semana. A identificação de riscos potenciais ou reais para a vida da criança permite, ao enfermeiro, planejar assistência individual e qualificada. A partir de então, intervenções podem ser efetivadas no sentido de aprimorar o cuidado em saúde e, consequentemente, a qualidade de vida das crianças, com redução das mortes infantis.

Descritores: Mortalidade Infantil; Assistência Perinatal; Enfermagem Pediátrica.

\section{ABSTRACT}

The present study aims at analyzing the child mortality profile Ribeirão Preto-SP inhabitants between 2009 and 2011 , based on data from the Mother-Infant Mortality Committee. In this period, there were 224 deaths, of which 58 were avoidable. There were more deaths among children under a week old, with low birth weight and gestational age under 30 weeks; pre-natal care was considered insufficient in most cases. There was an association between age of death and gestational age; increased years of schooling proved to be a protective factor regarding deaths in the first week of life. The identification of real or potential risks to the child's life allows nursing professionals to plan individual and qualified assistance. Such planning allows interventions to be carried out, in the sense of improving health care and consequently children's quality of life, reducing infant mortality.

Descriptors: Infant Mortality; Perinatal Care; Pediatric Nursing.

\section{RESUMEN}

Se objetivó analizar el perfil de la mortalidad infantil de residentes en Ribeirão Preto-SP entre 2009 y 2011 , a partir de los datos del Comité de Mortalidad Maternoinfantil. Durante el período, ocurrieron 224 fallecimientos, siendo 58 de ellos evitables. Niños con menos de una semana de vida, bajo peso al nacer y edad gestacional inferior a 30 semanas fueron las causas que presentaron más decesos; el prenatal fue considerado insuficiente en la mayoría de los casos. Existió asociación entre edad del fallecimiento y edad gestacional; tener mayor escolarización demostró un efecto protector en relación al fallecimiento en la primera semana. La identificación de riesgos potenciales o reales para la vida del niño le permite al enfermero planificar atención individual y calificada. Partiéndose de ello, pueden realizarse intervenciones apuntando a optimizar el cuidado en salud y, consecuentemente, la calidad de vida de los niños, con reducción de la mortalidad infantil.

Descriptores: Mortalidade Infantil; Atención Perinatal; Enfermería Pediátrica. 


\section{INTRODUÇÃO}

A redução da mortalidade infantil ainda se configura como desafio para os serviços de saúde, como também para a sociedade como um todo(1). Nesse sentido, instituições nacionais e internacionais se mobilizam traçando políticas de atenção à saúde que buscam soluções para o enfrentamento, como a exemplo do compromisso, de diversos países, com as Metas do Desenvolvimento do Milênio. Tais metas visam o alcance de patamares mais dignos de vida para a população, tendo como quarto objetivo, a redução da mortalidade infantil. Ao se considerar as políticas públicas de saúde brasileiras, é importante destacar ações como o Pacto pela Redução da Mortalidade Materna e Infantil(2); a Agenda de compromisso para atenção integral da criança e redução da mortalidade infantil ${ }^{(3)}$ e a Rede Cegonha ${ }^{(4)}$.

Os resultados divulgados pelo Censo 2010 apontam que no Brasil, no período 2000 a 2010, a taxa de óbito de crianças menores de um ano caiu de 29,7 para 15,6 por mil nascidos vivos (/1000NV), um decréscimo de $47,6 \%$ da taxa brasileira de mortalidade infantil( ${ }^{(5)}$. A região nordeste, com concentração histórica de elevados índices, apresentou uma expressiva redução. Em 2000, a taxa de mortalidade infantil (TMI) era de 41,6 óbitos/1000NV; em 2008, 21,7 óbitos/1000NV(6). Relatório do Fundo das Nações Unidas para a Infância (UNICEF) destaca o avanço global na redução das mortes infantis, ressaltando a não existência de empecilhos para progressos nessa diminuição, quer seja devido à localização geográfica de um país, quer seja relacionado à sua situação econômica(7). Apesar de o Brasil ocupar a posição 107 na tabela de morte de crianças, o mesmo destaca-se por ter sido responsável por uma redução de $73 \%$ da mortalidade nessa faixa etária, bem acima da média mundial, que foi de pouco mais de $40 \%$. Em 1990, o país registrava 58 óbitos/1000NV, reduzindo este valor para menos de 16 mortes por 1.000 crianças nascidas vivas no ano de $2011^{(7)}$.

Essa queda da mortalidade infantil no país está associada a diversas melhorias nas condições de vida e de atenção à saúde da criança, com relação a questões como alimentação e nutrição, saneamento básico, vacinação e modelo de atenção à saúde ${ }^{(8-9)}$. As melhorias na saúde de mães e crianças no país evidenciam como o Brasil evoluiu em termos de sistemas de saúde, condições de saúde e determinantes sociais ${ }^{(8)}$, transformando-se em sociedade predominantemente urbana, com taxa de fecundidade reduzida, educação primária universalizada, expectativa de vida ao nascer aumentada em cerca de cinco anos por década ${ }^{(9)}$.
Apesar da melhora da TMI, nacionalmente existem grandes desafios a serem superados, como as desigualdades regionais e as iniquidades relacionadas a grupos sociais específicos, para se aproximar dos níveis das regiões mais desenvolvidas do mundo, com cerca de cinco óbitos para cada mil nascidos $\operatorname{vivos}^{(9)}$. As desigualdades regionais das condições de saúde estão relacionadas com a mortalidade neonatal no Brasil, especialmente devido à falta de acesso aos serviços de saúde voltados para a saúde materna e infantil(10).

Para o estado de São Paulo, os dados da Rede Interagencial de Informações para a Saúde (RIPSA) demonstram queda; em 2000, a TMI era de 17,3 óbitos/1000nv, em 2009, 12,4 óbitos/1000NV, já nos anos de 2010 e 2011, a TMI representou 12,0 e 11,6 óbitos/1000NV, respectivamente. Com relação à taxa de mortalidade neonatal precoce, os dados também apontam redução no decorrer desse período. Em 2000, a taxa foi de 8,9 óbitos/1000NV, em 2009, 6,1 óbitos/1000NV, já em 2011 a taxa foi de 5,7 óbitos/1000NV ${ }^{(6)}$.

Dados da Secretaria Municipal de Saúde de Ribeirão Preto-SP (SMS-RP) demonstram esta redução na série histórica da mortalidade infantil em residentes no município $^{(11)}$. No ano de 2000 a TMI era de 12,9 óbitos/1000NV; em 2005, 10,93 óbitos/1000NV e em 2009 a TMI do município foi de 8,6 óbitos/1000NV. Para 2010 e 2011, os valores dessa taxa foram de 9,4 óbitos/1000NV e 9,7 óbitos/1000NV, respectivamente. No ano de 2012 houve queda, contabilizando 8,6 óbitos/1000NV.

Em 2000 os óbitos de menores de um mês de vida, no município, representaram 56,5\% (61) do total de mortes de menores de um ano (108). Para o ano de 2005 foram 64 (77\%) óbitos para um total de 83 nessa faixa etária. Em 2009, 75\% (51) do total de óbitos em menores de um ano foram de crianças com menos de um mês de vida ${ }^{(11)}$. No que diz respeito aos Coeficientes de Mortalidade Neonatal (CMNN) precoce, o município apresentou variações ao longo do tempo. Os CMNN para 2000 foram 8,48 óbitos/1000NV; em 2005, 8,43 óbitos/1000NV; em 2009, 6,5 óbitos/1000NV. No ano de 2010 o CMNN apresentou 5,2 óbitos/1000NV; em 2011 6,0 óbitos/1000NV e em 2012, 5,0 óbitos/1000NV, mostrando redução ao longo do tempo ${ }^{(11)}$.

No sentido de prover uma atenção integrada e voltada para os aspectos importantes da saúde infantil, há que se considerar a vigilância de óbitos infantis e sua respectiva investigação, como uma das prioridades do Ministério da Saúde ${ }^{(12)}$. Esta por sua vez, contribui para o 
cumprimento dos compromissos assumidos em defesa da saúde da criança.

Investigar tais mortes configura-se, portanto, em importante estratégia para a redução da mortalidade infantil e neonatal; contribui para a melhoria do registro de óbitos e possibilita que medidas de prevenção desses óbitos sejam adotadas nos serviços de saúde que atendem esta clientela ${ }^{(12)}$.

Sendo assim, a investigação dos óbitos infantis aliada à avaliação da assistência prestada a esta clientela nos serviços de saúde apresenta-se como de extrema importância, uma vez que possibilita a identificação de fortalezas e fragilidades que permitem a manutenção ou adequação necessárias para aprimoramento dos serviços.

É necessário acompanhar os complexos processos que envolvem a mortalidade infantil, mas não somente na busca por informações que constam em banco de dados nacionais, é importante também a busca por informações que permitam a análise da situação local da saúde e das ações que estão sendo realizadas, para identificar onde a intervenção deve ser realizada mais efetivamente $^{(13)}$. O conhecimento e a avaliação do número e situação das crianças que nasceram em um determinado local, possibilita a compreensão relacionada aos riscos que podem ocorrer nas condições de nascimento, crescimento e desenvolvimento dessas crianças $^{(14)}$.

A redução da mortalidade infantil, principalmente a neonatal, encontra-se vinculada ao reconhecimento de sua relevância pelos gestores do sistema de saúde. Oferecer visibilidade a esta situação constitui-se em importante passo com vista à tomada de decisões. Além disso, as ações em saúde contribuem para a melhoria das condições de vida e de saúde da criança, com reflexo na redução da mortalidade infantil ${ }^{(1,15)}$.

Nesse sentido, este estudo teve por objetivo analisar o perfil da mortalidade, nos anos de 2009, 2010 e 2011, de crianças menores de um ano residentes em Ribeirão Preto/SP, município de médio porte do interior paulista, referência regional e nacional para a assistência à saúde. Investigar tais óbitos possibilita a elaboração de indicadores de saúde que demonstrem a situação da atenção à saúde da mulher e da criança, contribuindo no planejamento do cuidado para redução das mortes infantis.

\section{MATERIAL E MÉTODOS}

Estudo transversal que envolveu a análise de todos os óbitos de crianças menores de um ano entre 2009 e
2011, investigados pelo Comitê de Mortalidade Materno Infantil da Secretaria Municipal de Saúde (SMS) e cuja evitabilidade foi classificada como evitável ${ }^{(12)}$.

Após classificação do óbito e de seu nível de evitabilidade e cumprindo seu papel educativo, o Comitê elabora uma carta a ser enviada ao serviço de saúde de referência da mãe e da criança em questão, esclarecendo os resultados discutidos no Comitê e indicando possibilidades de fortalecimento da atenção à saúde. Os dados obtidos de cada óbito são digitados em planilhas e arquivados na SMS. Além dessas planilhas, existem ainda registros extraídos dos prontuários acerca do percurso de cada mãe e bebê pelos serviços de saúde e de seus atendimentos.

Foi a partir dessas planilhas que se realizou a coleta de dados do presente estudo. Foram extraídas as informações tanto das gestantes quanto dos RN para compreensão do perfil da mortalidade infantil no município. Tais dados foram coletados no período de março a maio de 2012 e, posteriormente, tiveram entrada dupla em banco de dados. A análise pautou-se na estatística descritiva, com apresentação de frequências absolutas e relativas. Também é apresentada estatística inferencial, tendo sido considerada variável dependente, a idade do óbito, e variáveis independentes, idade e escolaridade maternas, pré-natal, Idade Gestacional (IG) e peso ao nascimento.

O projeto de pesquisa foi aprovado por Comitê de Ética em Pesquisa (protocolo no 1102/2009) e o sigilo dos dados coletados foi garantido, de acordo com as diretrizes que regulamentam as pesquisas envolvendo seres humanos.

\section{RESULTADOS}

A partir dos dados levantados junto ao Comitê de Mortalidade Materno Infantil, foi possível identificar que no período de 2009 a 2011, ocorreram 224 óbitos de menores de um ano, sendo que 58 (25,9\%) óbitos foram classificados como evitáveis. A Tabela 1 apresenta os nascidos vivos e as mortes ocorridas dentro do primeiro ano de vida no município.

Na Tabela 2 são apresentados os valores da TMI do município, por ano de ocorrência, comparadas às do estado de São Paulo e do Brasil.

Observa-se redução da TMI no decorrer dos anos, sendo que o município apresentou discreto aumento em 2011; porém, na média dos anos estudados, o município permanece com valor inferior, quando comparado ao estado e ao país. 
Tabela 1: Nascidos-vivos, óbitos em menores de um ano e taxa de mortalidade infantil, segundo ano de ocorrência. Ribeirão Preto, SP, Brasil, 2012.

\begin{tabular}{|c|c|c|c|}
\hline Ano de ocorrência & NV & Óbitos < 1 ano & TMI \\
\hline 2009 & 7822 & 67 & 8,6 \\
\hline 2010 & 8093 & 76 & 9,4 \\
\hline 2011 & 8353 & 81 & 9,7 \\
\hline Total & 24268 & 224 & $9,2 *$ \\
\hline
\end{tabular}

Notas: NV: nascido-vivo; * Média das TMI no período.

Tabela 2: Taxa de mortalidade infantil do município, estado de São Paulo e Brasil, segundo ano de ocorrência. Ribeirão Preto, SP, Brasil, 2012.

\begin{tabular}{|c|c|c|c|c|}
\hline \multirow{2}{*}{ Taxa de Mortalidade Infantil } & \multicolumn{4}{|c|}{ Ano de ocorrência } \\
\hline & 2009 & 2010 & 2011 & Média* \\
\hline Ribeirão Preto & 8,6 & 9,4 & 9,7 & 9,2 \\
\hline Estado de São Paulo & 12,4 & 12,0 & 11,6 & 12,0 \\
\hline Brasil & 16,8 & 16,0 & 15,3 & 16,0 \\
\hline
\end{tabular}

* Média das TMI no local de ocorrência e período do estudo.

Ao elencar as mortes por ano de ocorrência e evitabilidade, tem-se que dos 67 óbitos em 2009, 21 (31,3\%) foram classificados como evitáveis; em 2010, foram 76 óbitos, sendo 17 (22,4\%) evitáveis e em 2011, dos 81 óbitos, $20(24,7 \%)$ foram classificados como evitáveis.

Tendo em vista a melhoria do acesso aos serviços de saúde, a assistência no município é dividida em cinco Distritos de Saúde. Identificou-se o Distrito $4 \mathrm{com}$ maior ocorrência de casos, $16(27,6 \%)$ óbitos, seguido do Distrito 1 , com $14(24,1 \%)$ de casos. Dos 58 óbitos estudados, identificou-se $43(74,1 \%)$ crianças atendidas em hospitais vinculados ao SUS; os demais foram de crianças que nasceram em instituições hospitalares que atendem serviço complementar de saúde (convênios).

$\mathrm{Na}$ Tabela 3 encontram-se os dados referentes às variáveis maternas, idade e escolaridade e número de consulta pré-natal. Os achados apontam que $48,2 \%$ das mães têm idade inferior a 24 anos; 36,2\% possuem nove anos ou mais de estudo e $15,5 \%$ realizaram mais que seis consultas.

No que diz respeito à consulta pré-natal, importante ação para redução de óbitos infantis, 13 (22,4\%) mulheres não realizaram consulta ou tiveram esta informação não alimentada nas planilhas (dado ignorado). Além disso, para $12(20,7 \%)$ mulheres havia registro de realização da consulta; porém, sem informação do número total das mesmas.

Vinte e quatro $(41,4 \%)$ mulheres eram primigestas. Com relação aos demais óbitos, 11 (19\%) ocorreram na segunda gestação, oito $(13,8 \%)$ na terceira, sete $(12,1 \%)$ na quarta, dois $(3,4 \%)$ na quinta, três $(5,2 \%)$ na sexta, e três $(5,2 \%)$ na oitava gestação.

Tabela 3: Óbitos segundo a idade e escolaridade maternas e número de consultas pré-natal. Ribeirão Preto, SP, Brasil, 2012.

\begin{tabular}{|c|c|c|}
\hline \multirow{2}{*}{ Variáveis } & \multicolumn{2}{|c|}{ Óbitos } \\
\hline & $\mathbf{N}$ & $\%$ \\
\hline \multicolumn{3}{|l|}{ Idade da Mãe (anos) } \\
\hline 15 a 19 & 10 & 17,2 \\
\hline 20 a 24 & 18 & 31,0 \\
\hline 25 a 29 & 13 & 22,4 \\
\hline 30 a 34 & 09 & 15,5 \\
\hline 35 a 39 & 02 & 3,4 \\
\hline 40 a 44 & 04 & 6,9 \\
\hline 45 a 49 & 02 & 3,4 \\
\hline \multicolumn{3}{|l|}{ Escolaridade (anos de estudo) } \\
\hline$<05$ & 06 & 10,3 \\
\hline 05 a 08 & 13 & 22,4 \\
\hline 09 a 11 & 13 & 22,4 \\
\hline$>12$ & 08 & 13,8 \\
\hline Ignorado & 18 & 31,0 \\
\hline \multicolumn{3}{|l|}{ No de consultas de pré-natal } \\
\hline$<06$ & 24 & 41,4 \\
\hline 06 & 04 & 6,9 \\
\hline$>06$ & 05 & 8,6 \\
\hline Sem registro do $n^{\circ}$ de consultas & 12 & 20,7 \\
\hline Não realizou & 11 & 19,0 \\
\hline Ignorado & 02 & 3,4 \\
\hline Total & 58 & 100,0 \\
\hline
\end{tabular}


Apenas oito (13,8\%) gestantes foram encaminhadas ao pré-natal de alto risco; duas $(3,4 \%)$ gestantes tiveram esse dado ignorado na planilha.

A Tabela 4 apresenta as variáveis relacionadas ao RN. É possível identificar que $44(75,9 \%)$ óbitos ocorreram com IG menor que 37 semanas; dos que nasceram prematuramente, $27 \quad(61,4 \%)$ RN apresentaram prematuridade extrema.

Considerando o peso ao nascer, observa-se que $68,9 \%$ apresentaram baixo peso $(<2500 \mathrm{~g})$. Com relação à idade do óbito, houve maior concentração na primeira semana de vida $(56,9 \%)$.

As doenças maternas com maior registro foram bolsa rota prematura e trabalho de parto prematuro (TPP). Vinte e oito $(48,3 \%)$ mães apresentaram mais que uma doença registrada, como por exemplo: "vulvovaginite e TPP"; "infecção do trato urinário, bolsa rota e TPP"; "corioamnionite e bolsa rota". Também foram identificadas $13(22,4 \%)$ mães que não possuíam tal informação nas planilhas.

Considerando a causa de óbito, os dados apontam com maior frequência a prematuridade extrema ( $\mathrm{IG}<30$ semanas) e a Doença da Membrana Hialina (DMH), 27 $(46,5 \%)$ e nove $(15,5 \%)$ RN, respectivamente; 48 $(82,7 \%)$ RN apresentaram mais de uma causa de óbito, como por exemplo: "prematuridade extrema, $\mathrm{DMH}$ e pneumotórax"; "prematuridade e anóxia intrauterina".
Do total de óbitos, 37 (63,8\%) foram classificados como redutíveis por adequado controle na gravidez e sete $(12,1 \%)$ como redutíveis por adequada atenção ao parto; os demais foram classificados com mais de um nível.

Antes da realização das análises estatísticas, as variáveis de algumas categorias foram agrupadas (idade da mãe, escolaridade, número de consultas pré-natal e IG), no sentido de aumentar os tamanhos amostrais de cada estrato. Os valores classificados como "Ignorados" de todas as variáveis foram descartados das análises. Para a variável Pré-natal, a categoria "sem registro do número de consultas" também foi descartada, pois embora indique a presença de atendimentos, não indica a frequência de realizações dos mesmos.

A análise estatística foi dividida em duas etapas. $\mathrm{Na}$ primeira etapa, foram realizados testes de associação entre a variável Idade do óbito com as variáveis Idade da Mãe, Escolaridade, Pré-Natal, Idade Gestacional e Peso ao nascer. Nessa etapa foi realizado o teste exato de Fisher ${ }^{(16)}$. Apenas para as variáveis escolaridade (Tabela 5) e idade gestacional (Tabela 6), as associações apresentaram significância estatística, diferentemente das variáveis consultas pré-natal (Tabela 7) e peso ao nascimento (Tabela 8).

Tabela 4: Óbitos, segundo Idade Gestacional, peso ao nascer e idade de ocorrência. Ribeirão Preto, SP, Brasil, 2012.

\begin{tabular}{|c|c|c|}
\hline \multirow{2}{*}{ Variáveis } & \multicolumn{2}{|c|}{ Óbitos } \\
\hline & $\mathbf{N}$ & $\%$ \\
\hline \multicolumn{3}{|l|}{ Idade Gestacional } \\
\hline 22 a 29 semanas e 6 dias & 27 & 46,6 \\
\hline 30 a 34 semanas e 6 dias & 11 & 19,0 \\
\hline 35 a 36 semanas e 6 dias & 06 & 10,3 \\
\hline 37 semanas ou mais & 14 & 24,1 \\
\hline \multicolumn{3}{|l|}{ Peso ao nascer $(\mathbf{g})$} \\
\hline$<500$ & 02 & 3,4 \\
\hline $500-999$ & 23 & 39,7 \\
\hline $1000-1499$ & 06 & 10,3 \\
\hline $1500-1999$ & 04 & 6,9 \\
\hline $2000-2499$ & 05 & 8,6 \\
\hline $2500-2999$ & 08 & 13,8 \\
\hline$\geq 3000$ & 10 & 17,2 \\
\hline \multicolumn{3}{|l|}{ Idade do óbito (dias) } \\
\hline$\leq 06$ & 33 & 56,9 \\
\hline $07-27$ & 07 & 12,1 \\
\hline $28-364$ & 18 & 31,0 \\
\hline Total & 58 & 100,0 \\
\hline
\end{tabular}

Tabela 5: Análise univariada da idade do óbito, segundo escolaridade. Ribeirão Preto, SP, Brasil, 2012.

\begin{tabular}{|c|c|c|c|c|}
\hline & & \multicolumn{3}{|c|}{ Idade do óbito (dias) } \\
\hline & & $\leq 06$ & $>06$ & Total \\
\hline \multirow{3}{*}{ Escolaridade (anos de estudo) } & $\leq 08$ & 06 & 13 & 19 \\
\hline & $\geq 09$ & 16 & 05 & 21 \\
\hline & Total & 22 & 18 & 40 \\
\hline
\end{tabular}

Fisher $\mathrm{p}$-value $=0,01$ 
Tabela 6: Análise univariada da idade do óbito, segundo idade gestacional. Ribeirão Preto, SP, Brasil, 2012.

\begin{tabular}{|c|c|c|c|c|}
\hline & & \multicolumn{3}{|c|}{ Idade do óbito (dias) } \\
\hline & & $\leq 6$ & $>6$ & Total \\
\hline \multirow{4}{*}{ Idade Gestacional (semanas) } & $\geq 30 a<37$ & 6 & 11 & 17 \\
\hline & $\geq 37$ & 5 & 9 & 14 \\
\hline & $<30 s$ & 22 & 5 & 27 \\
\hline & Total & 33 & 25 & 58 \\
\hline
\end{tabular}

Fisher $\mathrm{p}$-value $=0.001543$

Tabela 7: Análise univariada da idade do óbito, segundo consultas pré-natal. Ribeirão Preto, SP, Brasil, 2012.

\begin{tabular}{|c|c|c|c|c|}
\hline & & \multicolumn{3}{|c|}{ Idade do óbito (dias) } \\
\hline & & $\leq 06$ & $>06$ & Total \\
\hline \multirow{3}{*}{ Pré-natal (consultas) } & $\geq 06$ & 04 & 05 & 09 \\
\hline & $<06$ & 19 & 16 & 35 \\
\hline & Total & 23 & 21 & 44 \\
\hline
\end{tabular}

Fisher $\mathrm{p}$-value $=0.7159$

Tabela 8: Análise univariada da idade do óbito, segundo peso ao nascimento. Ribeirão Preto, SP, Brasil, 2012.

\begin{tabular}{|c|c|c|c|c|}
\hline & & \multicolumn{3}{|c|}{ Idade do óbito (dias) } \\
\hline & & $\leq 6$ & $>6$ & Total \\
\hline \multirow{3}{*}{ Peso ao nascimento $(\mathrm{g})$} & $<2500$ & 26 & 14 & 40 \\
\hline & $\geq 2500 \mathrm{a} \leq 3000$ & 7 & 11 & 18 \\
\hline & Total & 33 & 25 & 58 \\
\hline
\end{tabular}

Fisher $p$-value $=0.08747$

Para as variáveis que foram encontradas associações, nos testes de Fisher, realizou-se análise de regressão logística(17) com a variável dependente, idade do óbito. Em todas as análises foi considerado um nível de significância de 5\% (alfa=0.05).

Já com relação à regressão logística, a Tabela 9 apresenta os resultados das estimativas dos parâmetros relacionados às categorias. Escolaridade $\geq 9$ anos, IG $\geq 37$ semanas e IG < 30 semanas. O intercepto é o nível de referência que representa escolaridade de até oito anos e IG de 30 a 36 semanas e seis dias. Apenas a categoria IG $\geq 37$ semanas não apresentou significância estatística com relação à IG de 30 a 36 semanas e seis dias.

Na Tabela 10, tem-se a probabilidade de óbitos para crianças com até seis dias de vida, preditas pelo modelo ajustado. Tem-se que para mães com escolaridade até oito anos e IG de 30 a 36 semanas e seis dias, a probabilidade de óbito da criança com até seis dias de vida é de 90,14\%; enquanto que mães com escolaridade maior que nove anos e IG de 30 a 36 semanas, a probabilidade de óbito de uma criança nessa faixa etária cai para $49,76 \%$.

Tabela 9: Estimação dos parâmetros do modelo logístico. Ribeirão Preto, SP, Brasil, 2012.

\begin{tabular}{|c|c|c|c|c|}
\hline Parâmetros & Estimativas & Erro Padrão & Estatística-t & p-valor \\
\hline (Intercepto) & 2,2131 & 1,0730 & 2,0626 & 0,0392 \\
\hline Escolaridade $>9$ anos & $-2,2225$ & 0,8922 & $-2,4910$ & 0,0127 \\
\hline IG $\geq 37$ semanas & $-0,1905$ & 1,1478 & $-0,1660$ & 0,8682 \\
\hline $\mathrm{IG}<30$ semanas & $-2,6216$ & 1,1434 & $-2,2929$ & 0,0219 \\
\hline
\end{tabular}

Tabela 10: Valores preditos de óbito na primeira semana de vida. Ribeirão Preto, SP, Brasil, 2012.

\begin{tabular}{cc} 
Parâmetros & Probabilidade de óbitos \\
(Intercepto) & 0,9014 \\
Escolaridade $\geq 9$ anos & 0,4976 \\
IG $\geq 37$ semanas & 0,8831 \\
IG $<30$ semanas & 0,3993 \\
Escolaridade $<9$ anos e IG $\geq 37$ semanas & 0,4502 \\
Escolaridade $<9$ anos e IG $<30$ semanas & 0,0672 \\
\hline
\end{tabular}

Outro resultado que chama atenção é a mãe ter IG acima de 37 semanas com escolaridade menor que oito anos de estudo; a criança tem $88,31 \%$ de probabilidade de morrer antes de seis dias. Ter mais anos de escolaridade, no presente estudo, demonstrou um efeito protetor com relação ao óbito na primeira semana de vida.

\section{DISCUSSÃO}

Os resultados obtidos a partir desse estudo são particularmente importantes, uma vez que dizem respeito a um município de médio porte, referência regional e nacional para atendimento em saúde, principalmente no setor hospitalar. O mesmo iniciou, há 
cerca de uma década, o Programa Saúde da Família, com progressão das Unidades de Saúde da Família por grande parte do município, além de uma rede de assistência à saúde organizada dentro de Distritos de Saúde. Tais Distritos possuem população definida a partir de aspectos geográficos, econômicos e sociais e agrupam várias unidades de saúde e outros equipamentos sociais.

A distribuição dos óbitos apontou o Distrito com a maior ocorrência, sendo que o mesmo apresenta grande concentração de moradias irregulares em áreas que não oferecem condições adequadas de saúde à população. Pesquisa desenvolvida, na década de 1990, no mesmo município, já apontava as regiões mais pobres respondendo com elevadas taxas de mortalidade infantil, quando comparadas àquelas mais privilegiadas ${ }^{(18)}$, o que se mantém no presente estudo, indicando que a renda ainda desempenha importante papel como determinante de saúde, apesar desses resultados contradizerem relatório do UNICEF já destacado anteriormente ${ }^{(7)}$.

As diferenças entre as regiões do país com relação à mortalidade infantil ainda precisam ser consideradas, uma vez que, muitas vezes, também dentro de um mesmo município, existem diversos estratos sociais. Fazse, portanto, importante identificar adequadamente os territórios com grupos populacionais sob alto risco de morte infantil, e até mesmo correlacionar os riscos para a mortalidade dessas crianças com variáveis socioeconômicas e de assistência à saúde ${ }^{(10)}$.

A ocorrência dos óbitos, em sua maior parte nos hospitais vinculados ao SUS, encontra suporte na referência de atendimento em saúde que estas instituições representam para a população do município, da região, e em alguns casos, do país. O município possui três hospitais vinculados ao SUS, sendo dois considerados como referência de atendimento de gestantes, partos e nascimentos de baixo e médio risco, e um deles, referência para atendimento de alto risco a essa clientela.

Nesse estudo, identificou-se que a maior parte das mães é jovem e com escolaridade relativamente alta, com mais de oito anos de estudo. Houve associação da variável escolaridade com o óbito, corroborando estudo(18) $^{(18)}$ em oposição a outro estudo(19) desenvolvido na região sul do país, que não identificou relação da variável escolaridade materna com a mortalidade infantil.

Apesar de o número de consultas de pré-natal não ter apresentado associação com o óbito no presente estudo, considera-se que esta variável permanece sendo de grande relevância ao se investigar os óbitos infantis ${ }^{(20)}$, uma vez que grande parte das gestantes realizou um número de consultas inferior ao recomendado pelo Ministério da Saúde e que cerca de um quinto delas não fez nenhuma consulta, como revelado em outros estudos ${ }^{(20-21)}$. As consultas de prénatal são claramente consideradas importantes para o acompanhamento do desenvolvimento saudável do feto, sendo consideradas fator de proteção para a mortalidade neonatal precoce ${ }^{(3,20)}$. Autores reforçam, em estudo, que o risco de mortalidade infantil esteve associado ao baixo número de consultas, configurando a importância do acompanhamento da mulher e do feto durante a gestação(22).

Com relação à Idade Gestacional e peso ao nascimento, identificou-se a maior parte dos nascimentos com menos de 34 semanas e peso ao nascimento menor que $2.500 \mathrm{~g}$, corroborando estudos $^{(21,23)}$ que apontam estas como sendo relevantes variáveis a serem dimensionadas, uma vez que podem representar maior risco para mortalidade neonatal ${ }^{(20,24)}$. No presente estudo, o risco de morrer na primeira semana de vida esteve associado ao tempo de gestação; porém, não esteve associado ao peso ao nascimento.

Identificou-se a ocorrência da maior parte dos óbitos na primeira semana de vida, corroborando com outras pesquisas $^{(20-21)}$. Mesmo havendo redução na mortalidade pós-neonatal, ainda prevalece a ocorrência de mortes dentro dos primeiros seis dias de vida, o que constitui problema de saúde pública e desencadeia grande investimento em políticas públicas de atenção à criança, conforme apontado anteriormente ${ }^{(2-4)}$.

Nesse sentido, faz-se necessário que ações sejam desenvolvidas para que haja redução dessas ocorrências, tais como aproximar as gestantes das unidades de saúde para início precoce do pré-natal e acompanhá-las não somente nas consultas, mas também disponibilizando equipes, com participação ativa do enfermeiro, para a realização de visitas domiciliares durante a gestação.

É preciso também atentar-se para que o RN chegue à unidade de saúde o mais precoce possível após o nascimento, com garantia de atendimento e seguimento nos serviços de saúde ${ }^{(25)}$. Nesse sentido, o município conta, há mais de 15 anos, com um programa de atenção ao RN que visa reduzir complicações para o binômio mãe-filho, com redução da mortalidade infantil, trabalhando na articulação entre maternidade e rede básica de saúde ${ }^{(25)}$.

Os resultados apontaram a incidência de patologias maternas no decorrer da gestação. Estudo demonstra relação estreita entre tal variável e a mortalidade infantil $^{(13)}$. Tais patologias podem desencadear o trabalho 
de parto antes do previsto, com nascimento prematuro, como nos dados da presente investigação. Com isso, esses bebês passam a apresentar comorbidades relacionadas à prematuridade ${ }^{(20,23)}$, o que pode levar a complicações e ter como desfecho o óbito.

Destacam-se ainda que, em algumas das variáveis analisadas, não foi possível obter dados completos, pela ausência dos mesmos nas planilhas do Comitê de Mortalidade Materno-Infantil. Uma vez que tais informações são retiradas de prontuários e relatórios médicos, como apontados anteriormente, as mesmas não podem ser negligenciadas, frente à sua relevância na predição das condições maternas, como algumas patologias que ocorrem durante a gestação, e que se encontram relacionadas às causas de óbito neonatal ${ }^{(23)}$. Entretanto, tais informações necessitariam estar completas para viabilizar uma análise mais acurada.

O nível de evitabilidade dos óbitos desse estudo condiz com o que a literatura revela ${ }^{(1-2,20,23)}$, ou seja, ainda são elevadas as mortes de crianças que poderiam ser reduzidas frente a um adequado controle na gestação. Esse controle envolve não somente as equipes que atuam junto à mulher, mas ela própria, uma vez que é preciso que também haja adesão da mesma aos cuidados preconizados pelos serviços de saúde no decorrer da gravidez.

Como fragilidade desse estudo, aponta-se a não investigação dos atendimentos realizados pelas gestantes nos serviços de saúde, a partir de informações dos prontuários de atendimentos. Esses são dados qualitativos e que poderiam responder às questões relativas ao cuidado ofertado na rede de atenção à saúde materno-infantil e à trajetória dessa clientela nesses serviços.

Cabe ressaltar ainda que, tanto o número de informações ignoradas referentes ao tempo de estudo quanto o número de consulta pré-natal não preenchido nas planilhas, fragilizaram a conclusão referente a estas variáveis no período estudado.

\section{CONCLUSÕES}

Dos óbitos evitáveis, avaliados nesse estudo, identificou-se a região de saúde do município que teve maior ocorrência de casos, com certa concentração de população com menor renda e algumas áreas ocupadas irregularmente. As crianças com menos de uma semana de vida, com baixo peso ao nascer e idade gestacional inferior a 37 semanas foram as que mais morreram; o pré-natal foi considerado não suficiente para a grande maioria dos casos investigados.
Esses dados apontam para a necessidade de ampliar o olhar para a atenção materno-infantil, buscando alternativas que viabilizem assistência cada vez mais qualificada à gestante e ao neonato. É preciso também compreender as questões que envolvem a não realização do pré-natal, ou a não adesão aos atendimentos ofertados nos serviços de saúde.

Os caminhos para o atendimento com qualidade estão traçados dentro das políticas públicas brasileiras de atenção à saúde materno-infantil. No cotidiano do trabalho, cabe a cada um dos profissionais de saúde, refletir sobre as fortalezas existentes, no sentido de mantê-las e verificar, dentro das dificuldades apresentadas, aquelas passíveis de modificação para redução da mortalidade infantil.

A equipe de saúde que atende mulheres e crianças, principalmente na atenção básica, além dos esforços habituais para renovar a compreensão das mulheres sobre a importância de um seguimento adequado no decorrer do pré-natal, necessita estar imbuída de um pensamento que se traduza na prática do acolhimento e da escuta dessa mulher.

Nesse sentido, o enfermeiro possui potencial para aprimorar esse modo de cuidar do outro e disseminar tal prática entre a equipe de saúde que atende a mulher e a criança. Este profissional de saúde acompanha a mulher, na rede básica de saúde, no decorrer de seu ciclo de vida, estando com a mesma no início da gestação, em seu primeiro atendimento. $O$ enfermeiro também precisa atentar-se para identificar situações que possam interferir negativamente na gravidez e, quando estas forem passíveis de intervenção, discutir junto à equipe estratégias de cuidado que viabilizem uma atenção qualificada à gestante e ao feto ou à mulher e à criança.

Este estudo permitiu apontar que a investigação de óbitos infantis se faz relevante na gestão do cuidado à saúde da criança. A identificação de riscos potenciais ou reais para a vida da criança permite, ao enfermeiro, planejar assistência individual e qualificada. A partir de então, intervenções podem ser efetivadas, no sentido de aprimorar o cuidado em saúde e consequentemente a qualidade de vida das crianças, com redução das mortes infantis. 


\section{REFERÊNCIAS}

1. Victora CG, Aquino EML, Leal MC, Monteiro CA, Barros FC, Szwarcwald CL. Maternal and child health in Brazil: progress and challenges. Lancet. 2011;377(9780):1863-76.

2. Ministério da Saúde. Secretaria de Atenção à Saúde. Departamento de Ações Programáticas Estratégicas. Pacto Nacional pela Redução da Mortalidade Materna e Neonatal. Brasília: Ministério da Saúde, 2004.

3. Ministério da Saúde. Secretaria de Atenção à Saúde. Departamento de Ações Programáticas Estratégicas. Agenda de compromissos para a saúde integral da criança redução da mortalidade infantil. Brasília, 2004.

4. Conselho Nacional de Saúde. Ministério da Saúde. Portaria no 650/2011-Planos de Ação regional e municipal da Rede Cegonha. Brasília (Brasil): Ministério da Saúde; 2011.

5. Instituto Brasileiro de Geografia e Estatística [Internet]. Brasília: Ministério da Saúde (BR) [cited 2012 aug 08]. Censo 2010. Available from: http://www.censo2010.ibge.gov.br/. 6. Rede Interagencial de Informações para a Saúde - RIPSA. Brasília: Ministério da Saúde (BR). [cited 2014 feb 04]. Indicadores e dados básicos-Brasil-2012. Available from: http://tabnet.datasus.gov.br/cgi/idb2012/matriz.htm. 7. Fundo das Nações Unidas para a Infância (UNICEF). Situação Mundial da infância. 2012.

8. Victora CG, Barreto ML, Leal MC, Monteiro CA, Schmidt MI, Paim $\mathrm{J}$ et al. Health conditions and health-policy innovations in Brazil: the way forward. Lancet. 2011;377(9782);2042-53. 9. Paim J, Travassos C, Almeida C, Bahia L, Macinko J. The Brazilian health system: history, advances, and challenges. Lancet. 2011;377(9779):1778-97.

10. Oliveira GS, Lima MCBM, Lira CO, Oliveira AGRC, Ferreira MAF. Desigualdade espacial da mortalidade neonatal no Brasil: 2006 a 2010. Ciênc Saúde Colet. 2013;18(8):2431-2441.

11. Secretaria Municipal da Saúde [Internet]. Ribeirão Preto. Estatísticas Vitais. [cited 2014 feb 04]. Nascimentos e óbitos. Available from: http://www.coderp.com.br/cgibin/dh?tabnet26/sim/obit2000.def.

12. Ministério da Saúde. Manual de Vigilância do óbito infantil e fetal e do comitê de prevenção do óbito infantil e fetal. Brasília: Ministério da Saúde, 2009.

13. Santana IP, Santos JM, Costa JR, Oliveira RR, Orlandi MHF, Mathias TAF. Aspectos da mortalidade infantil, conforme informações da investigação do óbito. Acta Paul Enferm. $2011 ; 24(4): 556-62$.

14. Ramos HAC, Cuman RKN. Fatores de risco para prematuridade: pesquisa documental. Esc. Anna Nery, 2009; 13(2):297-304.

15. Barros AJD, Ronsmans C, Axelson H, Loaiza E, Bertoldi AD, França GVA et al. Equity in maternal, newborn, and child health interventions in Countdown to 2015: a retrospective review of survey data from 54 countries. Lancet. 2012;379(9822):122533.

16. Mehta CR, Patel N R. A Network Algorithm for Performing Fisher's Exact Test in $\mathrm{r}$ x c Contingency Tables. J of Am Statistical Assoc. 1983; 78(382): 427-434.

17. Agresti A. An introduction to categorical data analysis. Second edition. New York: Wiley, 2007.

18. Goldani MZ, Barbieri MA, Bettiol H, Barbieri MR, Tomkins A.

Infant mortality rates according to socioeconomic status in a

Brazilian city. Rev Saúde Pública. 2001;35(3):256-61.

19. Doldan RD, Costa JSD, Nunes, MF. Fatores associados à mortalidade infantil em Foz do Iguaçu-PR. Epidemiol. Serv. Saúde. 2011;20(4):491-498.

20. Soares ES, Menezes GMS. Fatores associados à mortalidade neonatal precoce: análise de situação no nível local. Epidemiol. Serv. Saúde, Brasília. 2010;19(1):51-60.

21. Geib LTC, Fréu CM, Brandão M, Nunes ML. Determinantes sociais e biológicos da mortalidade infantil em coorte de base populacional em Passo Fundo, Rio Grande do Sul.

Ciênc Saúde Colet. 2010;15(2):363-370.

22. Maia LTS, Souza WV, Mendes ACG. Diferenciais nos fatores de risco para a mortalidade infantil em cinco cidades brasileiras: um estudo de caso-controle com base no SIM e no SINASC. Cad Saúde Públ. 2012; 28(11):2163-2176.
23. Vanderlei LCM, Simões FTPA, Vidal AS, Frias PG. Avaliação de preditores do óbito neonatal em uma série histórica de nascidos vivos no Nordeste brasileiro. Rev. Bras. Saúde Matern. Infant. 2010;10(4):449-458.

24. Salge AKM, Vieira AVC, Aguiar AKA, Lobo SF, Xavier RM, Zatta LT et al. Fatores maternos e neonatais associados à prematuridade. Rev. Eletr. Enf. [Internet]. 2009;11(3):642-6. Available from: http://www.fen.ufg.br/revista/v11/n3/v11n3a23.htm. 25. Furtado MCC, Mello DF, Parada CMGL, Pinto IC, Reis MCG, Scochi CGS. Avaliação da atenção ao recém-nascido na articulação entre maternidade e rede básica de saúde. Rev. Eletr. Enf. [Internet]. 2010;12(4):640-6. Available from: http://www.fen.ufg.br/revista/v12/n4/v12n4a07.htm.

Artigo recebido em 26/09/2012.

Aprovado para publicação em 23/01/2014.

Artigo publicado em 30/06/2014. 